\title{
Effect of lattice defects on Hele-Shaw flow over an etched lattice
}

\author{
E. L. Decker, Jordi Ignés-Mullol,* A. Baratt, and J. V. Maher \\ Department of Physics and Astronomy, University of Pittsburgh, Pittsburgh, Pennsylvania 15260
}

(Received 20 November 1998; revised manuscript received 22 April 1999)

\begin{abstract}
We examine the patterns formed by injecting nitrogen gas into the center of a horizontal, radial Hele-Shaw cell filled with paraffin oil. We use smooth plates and etched plates with lattices having different amounts of defects $(0-10 \%)$. In all cases, a quantitative measure of the pattern ramification shows a regular trend with injection rate and cell gap, such that the dimensionless perimeter scales with the dimensionless time. By adding defects to the lattice, we observe increased branching in the pattern morphologies. However, even in this case, the scaling behavior persists. Only the prefactor of the scaling function shows a dependence on the defect density. For different lattice defect densities, we examine the nature of the different morphology phases. [S1063-651X(99)12508-X]

PACS number(s): 68.70.+w, 47.20.Gv, 47.54.+r, 47.20.Hw
\end{abstract}

\section{INTRODUCTION}

Many researchers have examined the patterns formed when one fluid is forced into another of higher viscosity between two closely spaced flat surfaces (a Hele-Shaw cell) $[1,2]$. If the otherwise flat surfaces have an imposed anisotropy (for instance, a periodic lattice imposed on one of the surfaces), driven fluid interfaces exhibit very complicated anisotropic viscous fingering patterns, and under some conditions dendritic patterns can be formed, reminiscent of classical dendrites observed in solidification [3-9]. To our knowledge, the effect of lattice defects in viscous fingering in an anisotropic Hele-Shaw cell has rarely been addressed $[4,6]$. One numerical simulation and an experimental study employed a porous medium with no gap and flow only within etched channels [4]. For a similar system there was a gap [6]. These studies varied the randomness in the channel widths and produced thinner dendritic fingers and enhanced side branching when compared to the case with constant channel width. This is consistent with our results and may be a general feature of dendritic growth in the presence of defects.

Some progress has been made in describing the morphology of the patterns formed in an anisotropic, radial HeleShaw cell and in relating observed morphology to variation of control parameters [3,5,7-9]. Theoretical work has explored the similar morphologies occurring in solidification $[10,11]$. Some studies have constructed morphology phase diagrams showing the types of patterns (phases) formed over different ranges of the control parameters [3,5,7,9]. In other studies, dendritic finger growth has been analyzed theoretically [11] and experimentally [8] and found in each case to exhibit a power-law relation between finger tip position and time. These same studies also show a successful scaling of the dendrites at all stages of growth to match a theoretical shape. Descriptions of anisotropic pattern growth explain the dendritic patterns produced at low driving force to be controlled by an effective anisotropic surface tension, while den-

\footnotetext{
*Present address: Department of Chemistry, Tulane University, New Orleans, LA 70118.
}

drites at high driving force, which grow along a different direction from those at low driving force, result from kinetic effects $[7,8,10]$. In between these surface-tension dendritic and kinetic dendritic regimes, a tip splitting viscous fingering regime results $[7,8,10]$. As discussed above, experiments exhibit these different morphological phases, and good agreement between experimental and theoretical dendrite shape and evolution have been achieved.

We report measurements made in a radial Hele-Shaw cell with smooth plates and in a series of anisotropic, radial HeleShaw cells wherein we vary the density of defects in the imposed lattice. We present quantitative analysis of pattern characteristics for all cases studied. We also discuss, qualitatively, the effect of lattice defects on the different morphology phases.

\section{EXPERIMENTAL DESCRIPTION}

Our horizontal, radial Hele-Shaw cell is formed by a smooth, rigid upper glass plate $(2.5 \mathrm{~cm}$ thick, $61 \mathrm{~cm}$ diameter) with a $6 \mathrm{~mm}$ diameter hole in the center, and a lower smooth glass plate of diameter $56 \mathrm{~cm}$ and thickness $1.25 \mathrm{~cm}$. The lower plate rests on a large square, $1.25 \mathrm{~cm}$ thick glass plate in a bath of heavy paraffin oil (Fisher Scientific, 01224). The oil has a viscosity of $1.7 \mathrm{P}$ and a surface tension of $31 \mathrm{dyn} / \mathrm{cm}$ at $24{ }^{\circ} \mathrm{C}$. Nitrogen gas is the less viscous fluid which displaces the paraffin oil during a flow realization. To introduce the nitrogen under controlled conditions, we fill a large reservoir (a metal box with 28 L capacity) with nitrogen to a relative pressure of 4 psi. Then using flow valves, we inject nitrogen from the reservoir at constant flow rate into the Hele-Shaw cell through the hole in the upper glass plate. The areal flow rate (or areal growth rate), $Q$, is obtained from a linear fit to the area, $A$, of the growing pattern versus time, $t$. During a flow realization, the evolution of the pattern is viewed with a CCD camera and videotaped with an $\mathrm{S}-\mathrm{VHS}$ recorder. The images are later digitized and computer analyzed.

To produce anisotropy in the cell, a circular circuit board (40 cm diameter) with an etched rectangular lattice is mounted onto the lower circular glass plate. The copper islands of the etched lattices are $0.07 \mathrm{~mm}$ thick and their lat- 


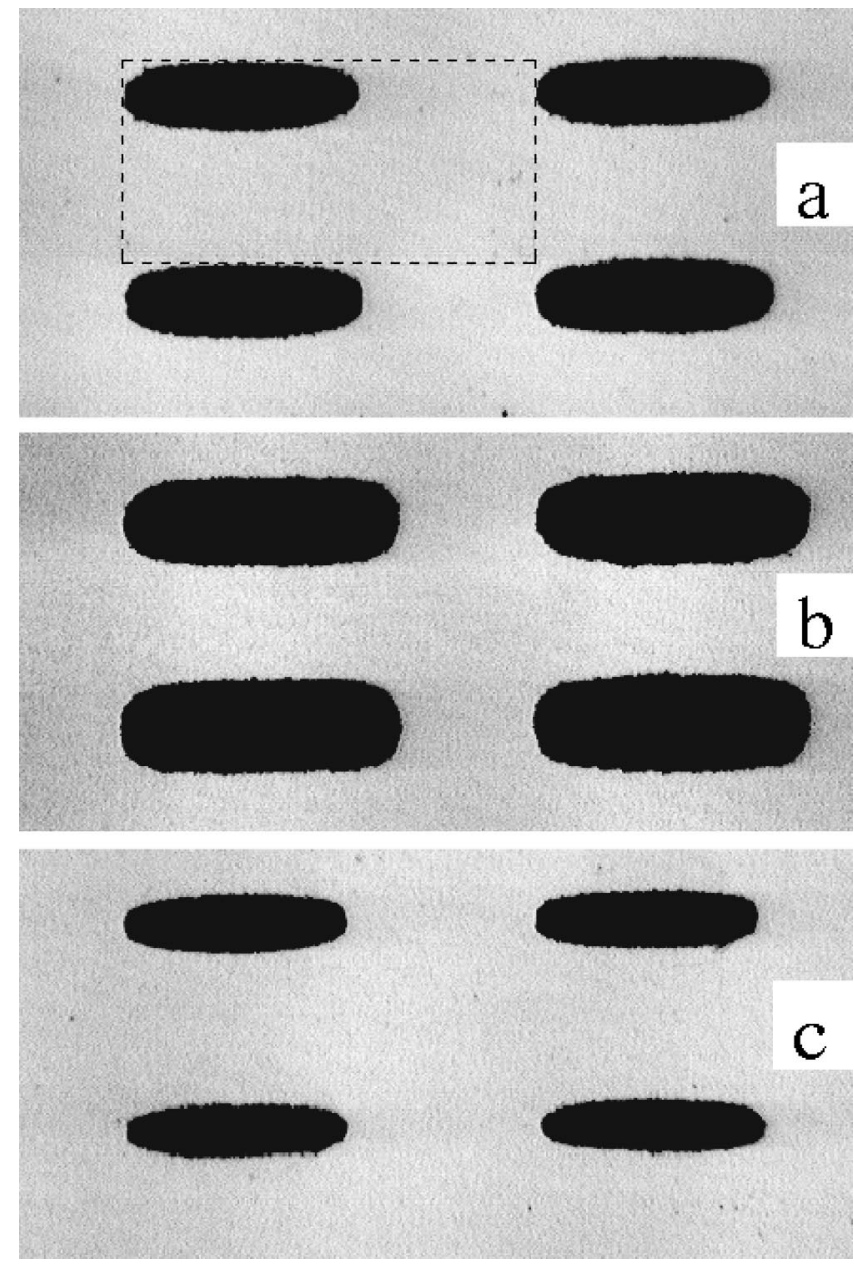

FIG. 1. Close-up view of each of the three etched circuit boards used. A section of each circuit board is shown, containing a unit cell of the lattice but no defects. (Defects are clearly seen in Fig. 2, where a larger section of each of the boards is shown.) Copper islands are $0.07 \mathrm{~mm}$ thick with lateral dimensions: (a) perfect lattice, $0.14 \times 0.47 \mathrm{~mm}$; (b) $3 \%$ defective lattice, $0.17 \times 0.55 \mathrm{~mm}$; (c) $10 \%$ defective lattice, $0.11 \times 0.46 \mathrm{~mm}$. The unit cell (shown by dashed lines) is the same for the three boards: $0.40 \times 0.80 \mathrm{~mm}$. Uncertainty in all lateral dimensions is $\pm 0.01 \mathrm{~mm}$.

eral dimensions are shown in Fig. 1. Three lattices in Fig. 1 show slightly different lateral dimensions for the etched copper islands (but not for the unit cell). Using lattices with no defects and with copper island variations even larger than those seen in Fig. 1, we have observed that these small differences in the regular parts of the etched plates do not alter our results significantly compared to the effects of lattice defects. (We do observe that the quality of the dendrites is somewhat sensitive to the details of the etched rectangular islands. However, the copper islands on our etched lattices were not circular enough for us to observe coexistence of morphology phases as reported by Banpurkar et al. [12].) Imperfect plates were produced by leaving defects (unetched copper regions) in the grooves between randomly selected copper islands. The percentage of defects for a given plate refers to the total area of unetched copper which forms the defects, relative to the total etched groove area on a perfect lattice with no defects. We report measurements with circuit boards with defect percentages of $0 \%$ (for the perfect lattice),

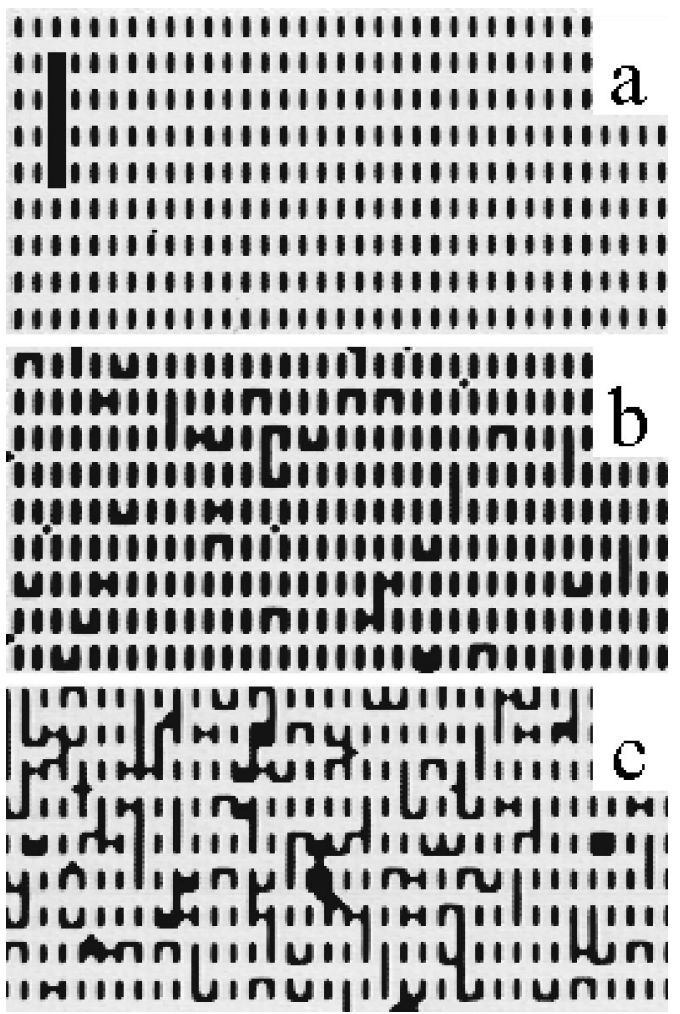

FIG. 2. Details of the etched lattice on the circuit boards with varying amounts of defects (a) $0 \%$, (b) $3 \%$, (c) $10 \%$. The vertical scale bar is $3 \mathrm{~mm}$ long. The dimensions of the copper islands are shown in Fig. 1.

3\%, and 10\%. (See Fig. 2.) We place Teflon spacers or strips of overhead transparency film between the upper glass plate and the circuit board (near the outer edge of the board) to set a gap of size $b$ between the plates. We report measurements for gap sizes of $b=0.11 \mathrm{~mm}, 0.22 \mathrm{~mm}, 0.38 \mathrm{~mm}, 0.79 \mathrm{~mm}$, and $1.59 \mathrm{~mm}$.

\section{RESULTS AND DISCUSSION}

Characterizations of tip position versus time and shapes for dendrites were calculated by Almgren et al. [11] and observed by Ignés-Mullol et al. [8] for the case of the perfect lattice. Evolution of dendrites with significant side branching (the type we observe with defective lattices) is not well suited to this type of scaling analysis. Dendrites produced with perfect lattices exhibit main, needlelike fingers growing along the lattice axes with increasing width and only minor side branching. Such dendrites can be scaled to match a characteristic dendrite shape [8]. However, with defective lattices, we observe (Sec. IIIC) dendrites which exhibit main fingers with nearly constant width and significant growth in the form of side branching. Thus, we cannot meaningfully apply the scaling analysis for smooth, needlelike dendrites to the more ramified, side branching dendrites produced with defective lattices. Rather, since defective lattices enhance side branching, we choose an analysis technique that quantifies the pattern ramification by measuring pattern perimeter $[13,14]$.

\section{A. Smooth plates}

Dimensional analysis of the isotropic flow equations for Hele-Shaw flow in a radial geometry gives a characteristic 

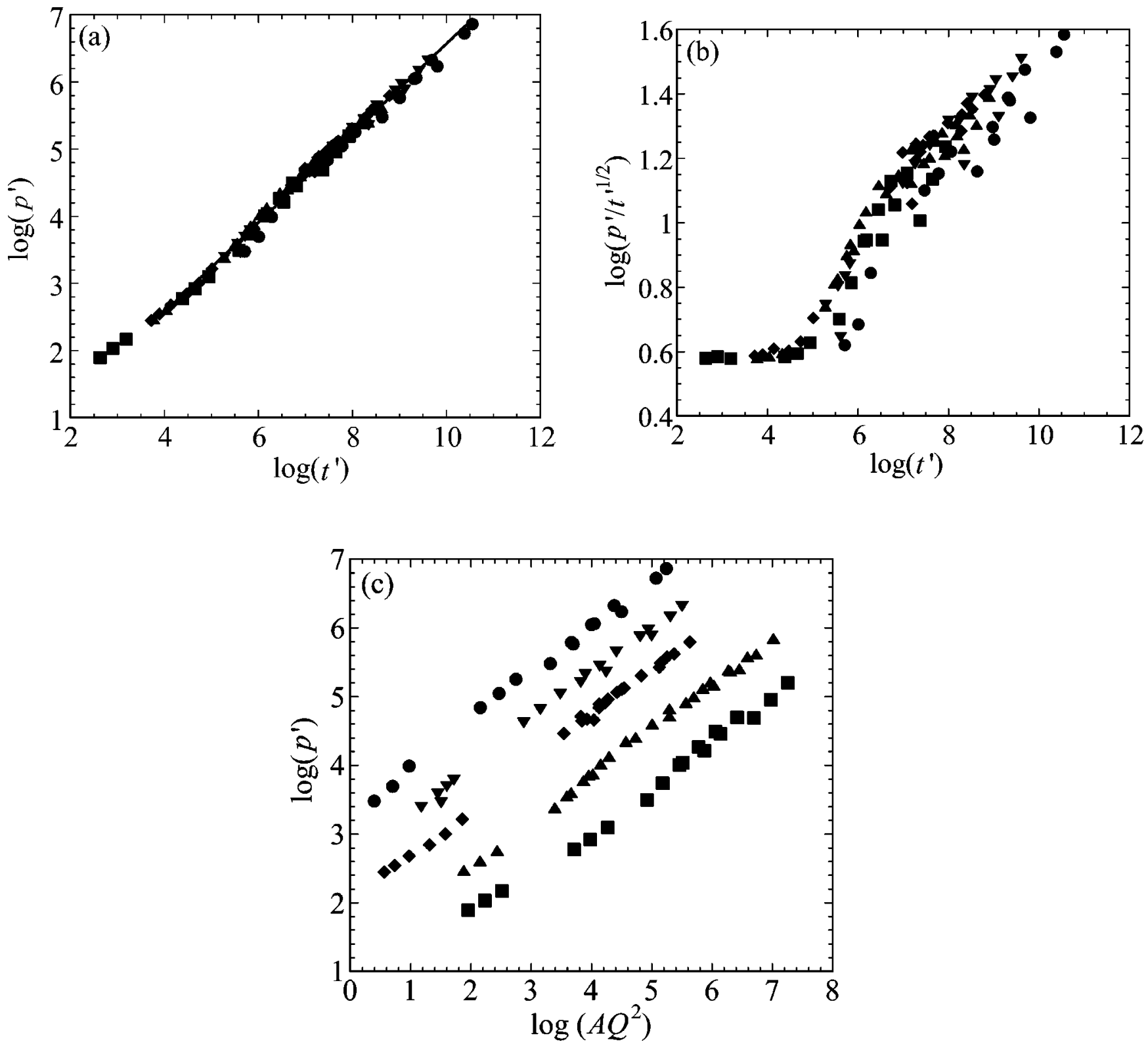

FIG. 3. Scaling of patterns produced between smooth plates, from many flow realizations at different flow rates $Q$, with three patterns (with different areas) from each flow realization, and at gaps of (circles) $b=0.11 \mathrm{~mm}$, (downward triangles) $b=0.22 \mathrm{~mm}$, (diamonds) $b$ $=0.38 \mathrm{~mm}$, (upward triangles) $b=0.79 \mathrm{~mm}$, (squares) $b=1.59 \mathrm{~mm}$. (a) $p^{\prime}$ plotted vs dimensionless time, $t^{\prime}$. A linear least-squares fit to the data for $\log \left(t^{\prime}\right)>4$ gives an average scaling exponent for $p^{\prime} \sim t^{\prime z}$ of $z=0.66 \pm 0.01$. (b) $p^{\prime} / t^{\prime 1 / 2}$ plotted vs $t^{\prime}$ clearly shows a compact-growth regime where $p^{\prime} / t^{\prime 1 / 2}$ is constant, and a regime of ramification where $p^{\prime} / t^{\prime 1 / 2}$ increases. (c) $p^{\prime}$ plotted vs $A Q^{2}$. Horizontal axis units are $\log \left(\mathrm{cm}^{4} / \mathrm{s}\right)$. Here, data from different gaps segregate into separate curves.

length, $L_{0}=b / \mathrm{Ca}$, and a characteristic time, $t_{0}=b^{2} /\left(Q \mathrm{Ca}^{2}\right)$, where $\mathrm{Ca}=\mu Q /(\sigma b)$ is the usual form of the capillary number in this type of flow with viscosity $\mu$, areal flow rate $Q$, surface tension $\sigma$, and gap $b[13]$. As we strive to develop meaningful quantitative measures of patterns, an interesting quantity is the perimeter, $p$, of a pattern, made dimensionless by $L_{0}\left(p^{\prime}=p / L_{0}\right)[13,14]$. As a growing pattern becomes unstable and tip splitting begins, the pattern becomes ramified. The perimeter of the pattern increases during the flow realization $\left(p^{\prime}\right.$ is a function of $\left.t^{\prime}\right)$. In Fig. 3(a), we plot the dimensionless perimeter, $p^{\prime}$, versus dimensionless time, $t^{\prime}$, for flow between smooth plates. The data shown include three patterns from each flow realization. Thus, we plot data, produced with different gap values, from patterns with dif- ferent areas (from different times during the flow) produced at the same flow rate, $Q$, as well as data from patterns produced at different flow rates. This shows that data from several runs at different values of $Q$ and $b$ all collapse to a single curve.

For our experimental setup, the initial area, $A_{0}$, is small compared to the area of the patterns from which area and perimeter data were extracted. Thus, since the area, $A$, of each pattern is $A=A_{0}+Q t \sim Q t$, then $t^{\prime}=t Q \mathrm{Ca}^{2} / b^{2}$ $\sim A \mathrm{Ca}^{2} / b^{2}=A / L_{0}^{2}=A^{\prime}$. We can compare the scaling behavior of our patterns to that of compact objects. For a compact growing object (such as a growing circle), the perimeter should increase as the square root of the area. Likewise, $p^{\prime}$ should increase as $t^{\prime 1 / 2}$. Careful inspection of the slope for 


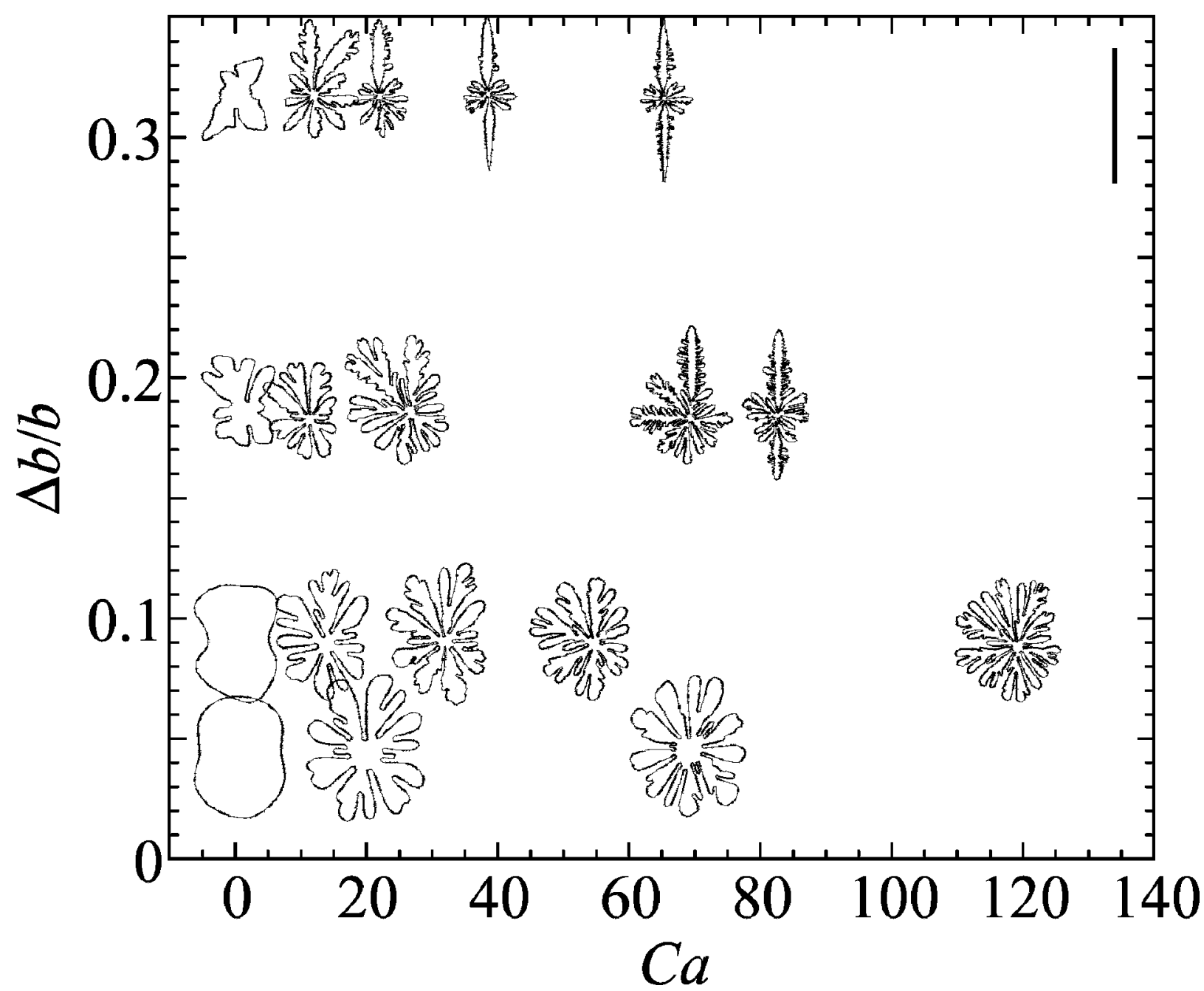

FIG. 4. Anisotropy parameter $\Delta b / b$ for data from four gaps $(b=0.22 \mathrm{~mm}, \Delta b / b=0.32 ; b=0.38 \mathrm{~mm}, \Delta b / b=0.18 ; b=0.79 \mathrm{~mm}$, $\Delta b / b=0.089 ; b=1.59 \mathrm{~mm}, \Delta b / b=0.044)$ plotted vs capillary number $\mathrm{Ca}=\mu Q /(\sigma b)$. Patterns from the end of flow realizations at the corresponding $\mathrm{Ca}$ are shown as data points on the plot. The vertical scale bar (showing the size of the patterns) is $25 \mathrm{~cm}$ long.

$\log \left(t^{\prime}\right)<4$ in Fig. 3(a) shows a slope of $\frac{1}{2}$. (Throughout this paper, $\log$ means $\log _{10}$.) Plotting $p^{\prime} / t^{\prime 1 / 2}$ versus $t^{\prime}$ in Fig. 3(b) very clearly reveals this compact-object growth for $\log \left(t^{\prime}\right)<4$. Patterns in this range of $t^{\prime}$ have minimal tip splitting and are often simple round shapes with constant $p^{\prime} / t^{\prime 1 / 2}$. For $\log \left(t^{\prime}\right)>4$, patterns become ramified and $p^{\prime} / t^{\prime 1 / 2}$ increases. The linear fit of $\log \left(p^{\prime}\right)$ vs $\log \left(t^{\prime}\right)$ [for $\left.\log \left(t^{\prime}\right)>4\right]$ in Fig. 3(a) shows a scaling exponent of $0.66 \pm 0.01$. Thus, within our range of pattern sizes where fingering occurs, the average scaling exponent differs significantly from the value of $\frac{1}{2}$ for compact objects.

In Fig. 3(c), plotting $p^{\prime}$ versus $A Q^{2}$ reveals similar scaling behavior as when plotting versus $t^{\prime}$. However, such a plot allows the gap dependence of the data to be manifest. In Fig. 3 (b), the data segregate into separate parallel curves for each gap. Thus, the perimeter increases at constant $A Q^{2}$ as the gap decreases.

\section{B. Perfect lattice}

At the larger gaps of $b=0.79 \mathrm{~mm}$ and $1.59 \mathrm{~mm}$, patterns produced over perfect (defect-free) etched lattices at low flow rates are smooth and round and patterns at our largest flow rates exhibit no dendrites. Thus, results at these larger gaps are similar to those with smooth plates. This observation is consistent with the calculations of Sarkar and Jasnow [15], where they predict the effects of the anisotropy to vanish when the anisotropy parameter falls below 0.07. Even though we are not confident of how to write an anisotropy parameter that relates to our experimental parameters, the strength of the anisotropy may be related to the depth of the grooves, $\Delta b$, relative to the gap, $b$. With $\Delta b=0.07 \mathrm{~mm}$, the above mentioned gaps give $\Delta b / b=0.089$ and 0.044 , respectively. At smaller gaps of $b=0.38 \mathrm{~mm}$ and $0.22 \mathrm{~mm}$, because of the enhanced influence of the lattice $(\Delta b / b=0.18$ and 0.32, respectively), we observe different morphological phases: surface tension dendrites at our lowest $\mathrm{Ca}$ (characterized by growth $45^{\circ}$ relative to the lattice axes) [8], tip splitting at low to intermediate $\mathrm{Ca}$, and kinetic dendrites at higher $\mathrm{Ca}$ (see Fig. 4). As reported previously for lattices with twofold symmetry [8], these kinetic dendrites grow from the injection hole along one axis of the lattice, with tip splitting fingering growing from the injection hole in other directions. Figure 4 implies that for increasing anisotropy, the transition from viscous fingering to kinetic dendrites moves to lower Ca.

Even though with the perfect lattice we can observe dendritic patterns at small gaps and no dendritic patterns at large 

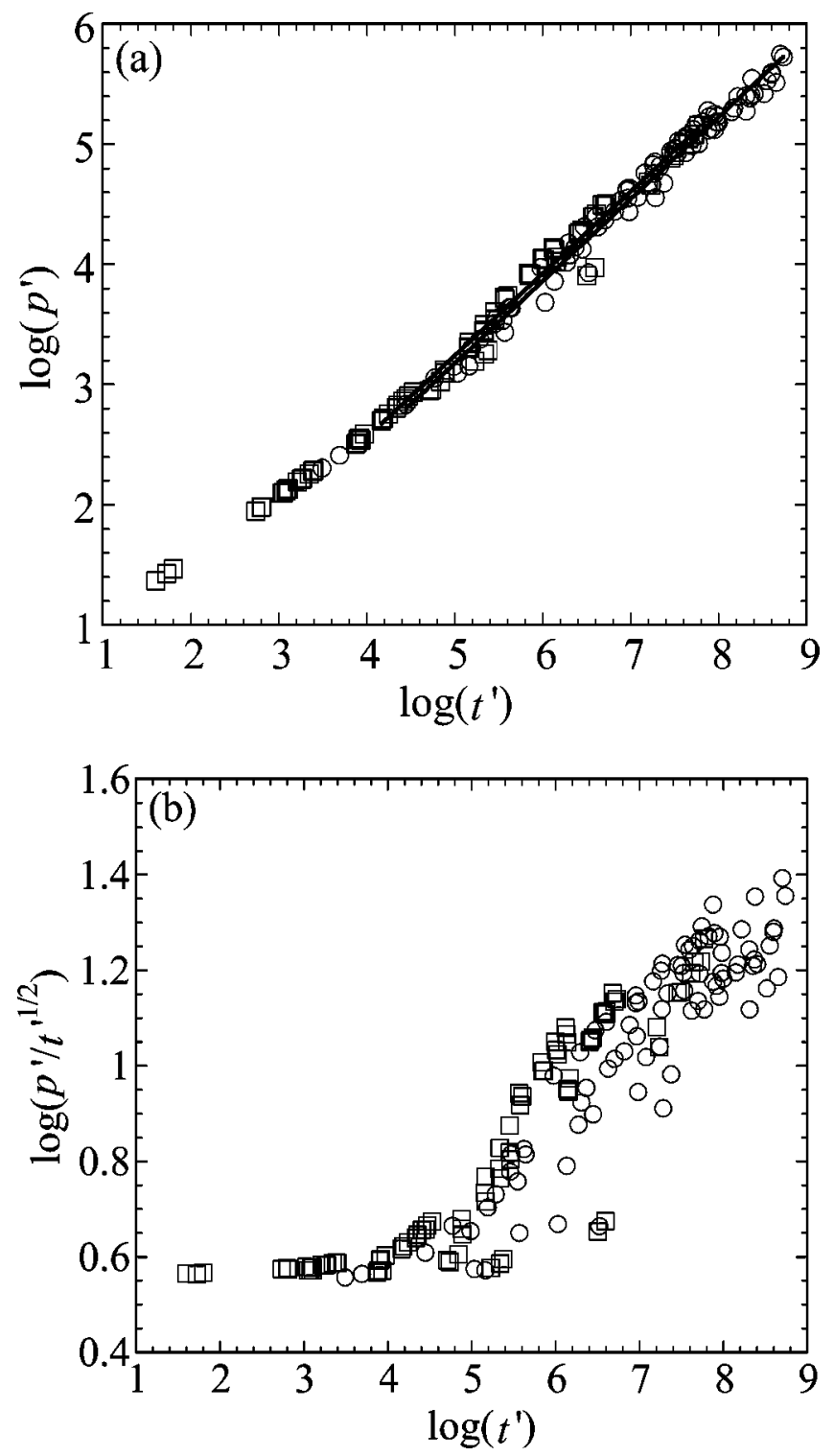

FIG. 5. Scaling of patterns produced with the perfect lattice (no defects) at two gaps. The data shown here come from many flow realizations at different flow rates $Q$, with five patterns (with different areas) from each flow realization. (Circles) $b=0.22 \mathrm{~mm}$, large anisotropy. (Squares) $b=1.59 \mathrm{~mm}$, small anisotropy. (a) $p^{\prime}$ plotted vs $t^{\prime}$ hardly distinguishes between cases of large anisotropy ( $b$ $=0.22 \mathrm{~mm})$, small anisotropy $(b=1.59 \mathrm{~mm})$, and no anisotropy [see Fig. 3(a)]. A three-parameter fit to both data sets simultaneously (see text) gives an average scaling exponent of $z=0.68$ \pm 0.01 for the scaling function $p^{\prime}=a t^{\prime z}$, and prefactors of $a$ $=0.58 \pm 0.08$ for $b=0.22 \mathrm{~mm}$, and $a=0.67 \pm 0.07$ for $b=1.59 \mathrm{~mm}$. (b) $p^{\prime} / t^{\prime 1 / 2}$ plotted vs $t^{\prime}$ still shows a compact-growth regime where $p^{\prime} / t^{\prime 1 / 2}$ is constant, and a regime of ramification where $p^{\prime} / t^{\prime 1 / 2}$ increases.

gaps, Figs. 5(a) and 5(b) show little distinction in the scaling behavior for isotropic and anisotropic growth. In Fig. 5 (a) we plot $p^{\prime}$ versus $t^{\prime}$ for patterns produced with the perfect lattice at a large gap (small anisotropy), $b=1.59 \mathrm{~mm}$, and at a small gap (large anisotropy), $b=0.22 \mathrm{~mm}$. Figure 5(b) shows that the transition from compact growth to ramified growth occurs near $\log \left(t^{\prime}\right)=4$ as it did with smooth plates. We perform a three-parameter fit to the data in Fig. 5(a) for $\log \left(t^{\prime}\right)>4$. In this fitting process, the slopes are forced to be the same, but the intercepts can differ. Thus, we fit $\log \left(p^{\prime}\right)$ $=\log \left(a_{0.22}\right)+z \log \left(t^{\prime}\right)$ and $\log \left(p^{\prime}\right)=\log \left(a_{1.59}\right)+z \log \left(t^{\prime}\right)$ simultaneously to the data sets for gaps of $b=0.22 \mathrm{~mm}$ and $b=1.59 \mathrm{~mm}$, respectively. Thus, the three parameters are $z$, $a_{0.22}$, and $a_{1.59}$. (This fit has a reduced $\chi^{2}$ that is about equal to the average of those obtained by fitting, two-parameter fits to each data set individually. However, the three-parameter fit reduces the effect of uncertainty in the individual slopes from washing out any trend in the intercepts.) The results show that the scaling exponent $(z=0.68 \pm 0.01)$ is not significantly different from that $(z=0.66 \pm 0.01)$ obtained with smooth plates. (In fact, the fingering patterns produced with perfect lattices at the four gaps we used give an average exponent of $z=0.67 \pm 0.01$.) The prefactors in the scaling function, $p^{\prime}=a t^{\prime z}$, show a nonsignificant dependence on the anisotropy ( $a=0.58 \pm 0.08$ for $b=0.22 \mathrm{~mm}$ and $a=0.67$ \pm 0.07 for $b=1.59 \mathrm{~mm}$ ). This insignificant offset of the curves is difficult to see in Fig. 5(a). Any difference is even difficult to observe in $p^{\prime} / t^{\prime 1 / 2}$. However, for $\log \left(t^{\prime}\right)>4$ in Fig. 5(b), $p^{\prime} / t^{\prime 1 / 2}$ does appear slightly smaller for the case with larger anisotropy. Thus, the scaling behavior we measure hardly distinguishes between pattern formation with smooth plates and that with etched lattices.

\section{Defective lattices}

To examine the effect of defects in our anisotropic HeleShaw cell, we use a small gap $(b=0.22 \mathrm{~mm})$ where the influence of the etched circuit board (lattice and defects) is strong. Figure 6(a) shows a comparison of $p^{\prime}$ versus $t^{\prime}$ for the perfect lattice, and for lattices with $3 \%$ and $10 \%$ defects. The data overlap significantly so that by eye it is difficult to distinguish any difference between the data from different defect densities. Figure 6(a) does show significant broadening of the curve in the $p^{\prime}$ direction compared to that seen in Fig. 3(a) and Fig. 5(a). In order to better observe the effect of the defects, we smoothed the data with a window of seven data points in Fig. 6(b). Here, three curves can be distinguished for each of the defect densities. By examining the data range where well-developed kinetic dendrites are observed [for $\log \left(t^{\prime}\right)>7$ ], the smoothed data definitely show that, for constant $t^{\prime}$, the perimeter increases as the defect density increases. In Fig. 6(c) we plot $p^{\prime} / t^{\prime 1 / 2}$ versus $t^{\prime}$. Here, we observe that the defective lattices produce ramified patterns even for $\log \left(t^{\prime}\right)<4$. This is seen mostly in the data from the $10 \%$ defective plate. In Fig. 6(d) we plot the same data but smoothed with an averaging window of seven data points. Here, we clearly observe increased ramification (as measured by $\left.p^{\prime} / t^{\prime 1 / 2}\right)$ in the dendritic-growth regimes $\left(t^{\prime}\right.$ $<5.5$ and $\left.t^{\prime}>7\right)$ as the defect density increases.

To further quantify the effect of the defects, in Fig. 6(a), we do a simultaneous, four-parameter fit to the three data sets (for $0 \%, 3 \%$, and $10 \%$ defects) over the entire range of $t^{\prime}$. We fit $\log \left(p^{\prime}\right)=\log \left(a_{0}\right)+z \log \left(t^{\prime}\right), \quad \log \left(p^{\prime}\right)=\log \left(a_{3}\right)$ $+z \log \left(t^{\prime}\right)$, and $\log \left(p^{\prime}\right)=\log \left(a_{10}\right)+z \log \left(t^{\prime}\right)$ simultaneously to the data sets for $0 \%, 3 \%$, and $10 \%$ respectively. Thus, the four parameters are $z, a_{0}, a_{3}$, and $a_{10}$. (This fit has a reduced $\chi^{2}$ that is about equal to the average of those obtained by fitting linear, two-parameter fits to each data set individually. However, the four-parameter fit reduces the effect of 

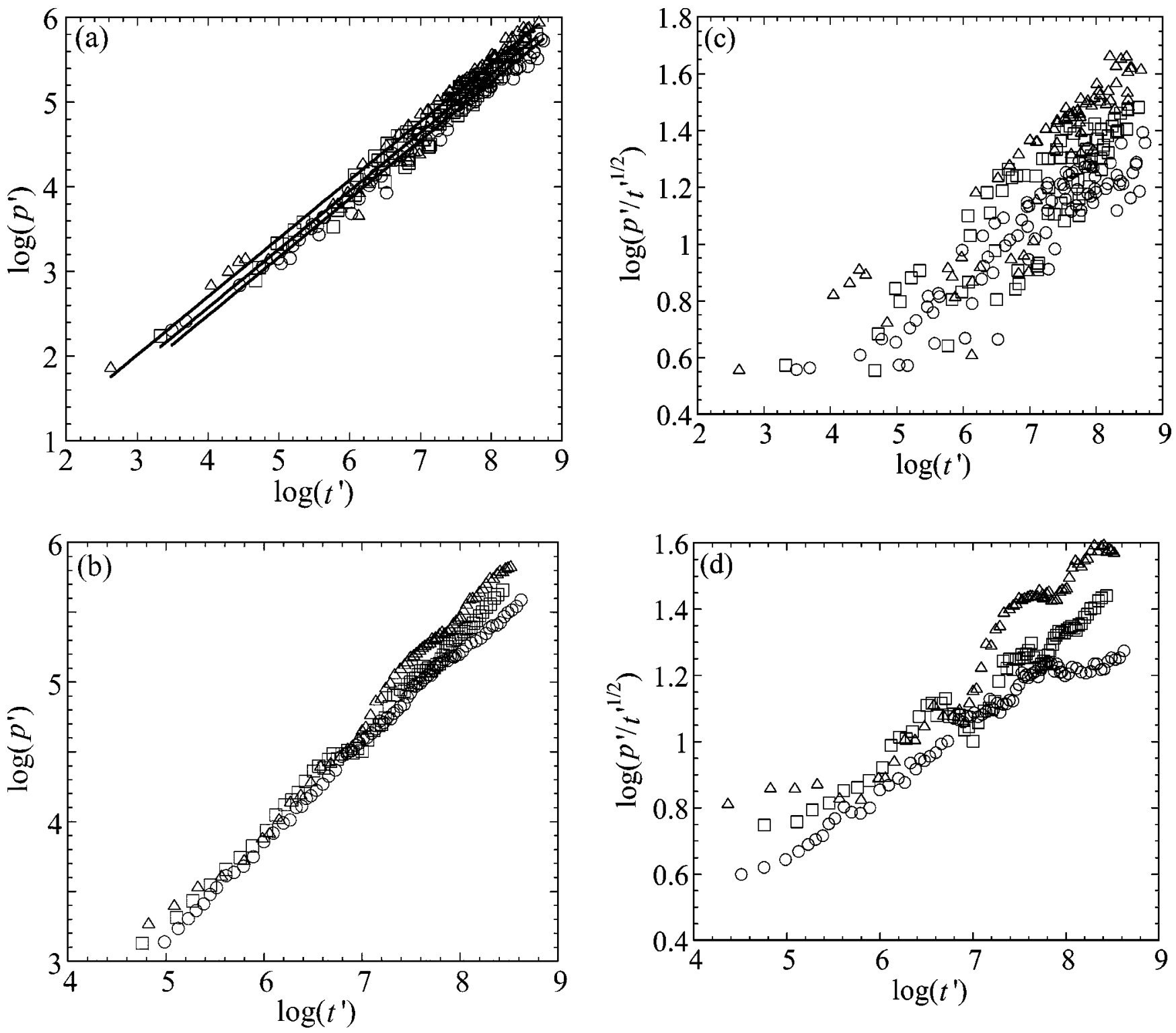

FIG. 6. Scaling of patterns produced with the lattices with (circles) $0 \%$ defects, (squares) 3\% defects, and (triangles) $10 \%$ defects. Data come from many flow realizations at different flow rates $Q$ at one gap, $b=0.22 \mathrm{~mm}$, with five patterns (with different areas) from each flow realization. (a) $p^{\prime}$ plotted vs $t^{\prime}$. The four-parameter, linear least-squares fit gives $z=0.69 \pm 0.01$ and (lowest line) $a=0.53$ for $0 \%$ defects, (middle line) $a=0.65$ for $3 \%$ defects, and (top line) $a=0.87$ for $10 \%$ defects, for the scaling function, $p^{\prime}=a t^{\prime z}$. (b) $p^{\prime}$ plotted vs $t^{\prime}$. The data are smoothed using an averaging window of seven data points. (c) $p^{\prime} / t^{\prime 1 / 2}$ plotted vs $t^{\prime}$ no longer shows a compact-growth regime where $p^{\prime} / t^{\prime 1 / 2}$ is constant for the defective plates. (d) $p^{\prime} / t^{\prime 1 / 2}$ plotted vs $t^{\prime}$. The data are smoothed using an averaging window of seven data points.

uncertainty in the individual slopes from washing out the trend in the intercepts that can be detected by examination of the data [see Figs. 6(b) and 6(d)].) Thus, for the scaling function, $p^{\prime}=a t^{\prime z}, z=0.69 \pm 0.01$, while $a$ depends on the density of defects $(a=0.52 \pm 0.06$ for $0 \%$ defects, $a=0.65$ \pm 0.07 for $3 \%$ defects, and $a=0.87 \pm 0.09$ for $10 \%$ defects).

As suggested by models of unstable interface growth in the presence of quenched disorder [16], the role of the defects might be thought of as a rescaling of an effective surface tension. For instance, the effect of the defects in the scaling function $p^{\prime}=a t^{\prime z}$, contained in the parameter $a$ (which depends on the amount of defects), can be absorbed into $t^{\prime}$. Recall that $t^{\prime}$ is proportional to $\sigma^{-2}$. This surface tension, $\sigma$, in $t^{\prime}$ can be replaced by an effective $\sigma^{\prime}$ $=\left(a_{0} / a\right)^{1 /(2 z)} \sigma$, such that for the perfect lattice $\sigma^{\prime}=\sigma$.
Thus, $\sigma^{\prime}$ decreases with increasing defect density. For instance, for $10 \%$ defects, $\sigma^{\prime}=0.7 \sigma$ (a 30\% decrease in the effective surface tension).

Figure 7 shows some patterns from which the perimeters were obtained for lattices with $0 \%, 3 \%$, and $10 \%$ defects. The patterns chosen for display here are those obtained at the end of flow realizations. We do not observe any clear shift in the transition points in $\mathrm{Ca}$ between different morphology phases. However, we do observe that the nature of these different morphology phases is drastically changed by the addition of defects. In all cases, the randomness of the patterns seems to increase as the defect density increases. For the surface tension dendritic pattern shown at the lowest values of $\mathrm{Ca}$ (see Fig. 7), the degree of definition of the dendritic tips seems to decrease as the defect density increases. 


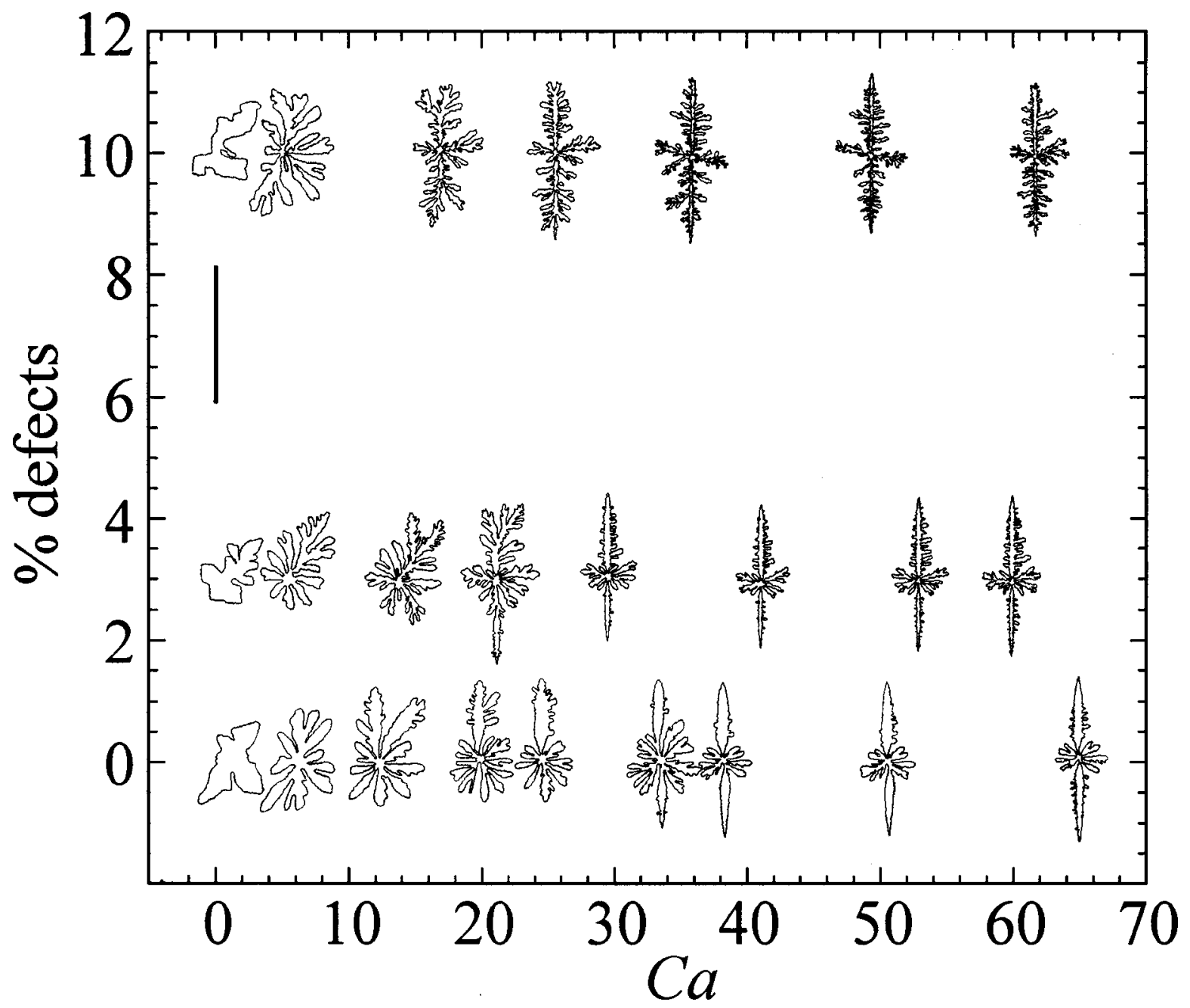

FIG. 7. Percentage of defects plotted vs capillary number $\mathrm{Ca}=\mu Q /(\sigma b)$. Patterns from the end of flow realizations at the corresponding $\mathrm{Ca}$ are shown as data points on the plot. The vertical scale bar (showing the size of the patterns) is $25 \mathrm{~cm}$ long.

At $10 \%$ defects, the "surface tension dendrite" has a rather random looking shape, as opposed to the more defined dendritic tip shape seen with the perfect lattice. We see the effects of lattice defects most prominently in the morphology of the kinetic dendrites. Kinetic dendrites produced with the perfect lattice can exhibit needle fingers with almost no side branching. We always observe significant side branching on kinetic dendrites produced with $3 \%$ or $10 \%$ defects. With $10 \%$ defects, the defect-enhanced ramification produces kinetic dendrites that vary significantly from the "classical" dendrite shape with regular side branching extrusions, which are observed with $0 \%$ and $3 \%$ defects.

We observe other specific qualitative features of kinetic dendrites which change with defect density. Figure 8 compares kinetic dendrites produced at roughly the same flow rate but with different lattice defect densities. Clearly, the finger width decreases and the side branching amplitude increases with increasing lattice defects.

\section{CONCLUSIONS}

We have reexamined the scaling of dimensionless perimeter, $p^{\prime}$, versus dimensionless time, $t^{\prime}$, for patterns produced in a radial Hele-Shaw cell. This scaling shows that, in general, as the gap, $b$, decreases and as the flow rate, $Q$, in-

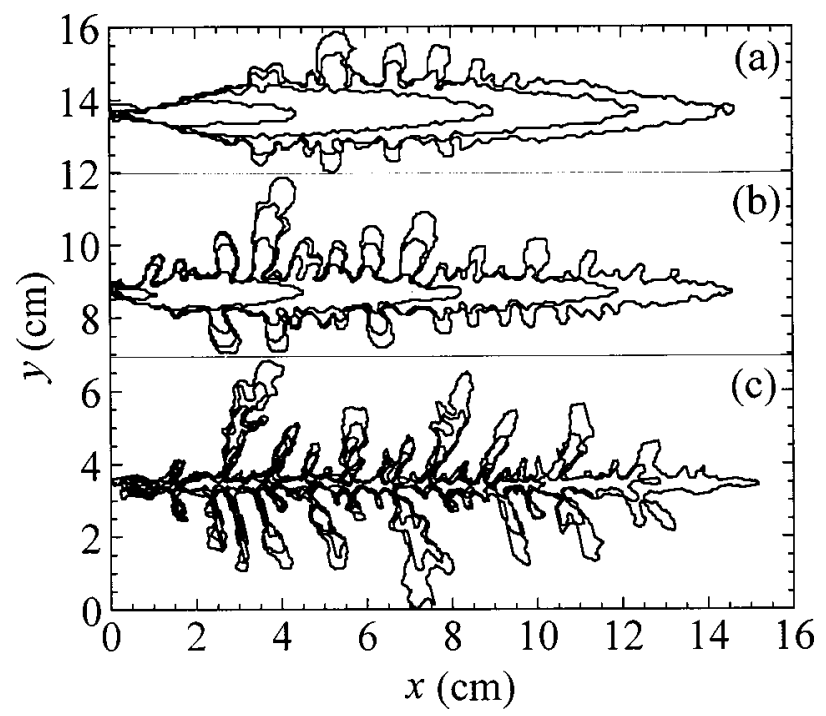

FIG. 8. Time series of patterns for development of dendrites: (a) $0 \%$ defects, areal flow rate for finger is $Q_{f}=11.7 \pm 0.4 \mathrm{~cm}^{2} / \mathrm{s}$; (b) $3 \%$ defects, $Q_{f}=10.9 \pm 0.5 \mathrm{~cm}^{2} / \mathrm{s}$; (c) $10 \%$ defects, $Q_{f}=11$ $\pm 2 \mathrm{~cm}^{2} / \mathrm{s}$. The time step between intermediate patterns for each finger is $0.6 \mathrm{~s}$. 
creases, patterns become more ramified. Patterns that grow as circular bubbles scale as compact objects with $p^{\prime} \sim t^{\prime z}$, with $z=0.5$. As bubbles begin to ramify by tip splitting or by dendritic growth with side branches, $z$ increases. Over our size range of fingering patterns produced with smooth plates, we measure an average exponent of $z=0.66 \pm 0.01$. Adding anisotropy in the form of an etched lattice does not significantly change this scaling behavior. In this case for fingering patterns, we measure an average exponent of $z=0.67 \pm 0.01$. Addition of lattice defects to the case with anisotropy gives an average exponent $(z=0.69 \pm 0.01)$ which is not significantly different from that for isotropic plates. The effect of the defects is to change the prefactor to the scaling function $p^{\prime}=a t^{\prime z}$. The prefactor, $a$, increases as the defect density increases. Thus, for patterns at constant $t^{\prime}$ the dimensionless perimeter increases as the defect density increases.
Defects to the imposed lattice in an anisotropic, radial Hele-Shaw cell have a dramatic effect on the resulting pattern morphology. The lattice defects tend to increase randomness in pattern ramification in all morphology phases. Thus, the nature of dendritic growth can appear quite different from that of classical dendrites produced with a perfect lattice. In the regime of kinetic dendrites, with dendritic growth along the direction of a lattice axis, the main needle fingers become thinner and the side branching amplitude increases as the defect density increases.

\section{ACKNOWLEDGMENTS}

We thank Dr. David Jasnow for his helpful comments. This work was supported by the U.S. Department of Energy under Grant No. DE-FG02-84ER45131.
[1] K. V. McCloud and J. V. Maher, Phys. Rep. 260, 139 (1995).

[2] P. Meakin, Fractals, Scaling and Growth Far From Equilibrium (Cambridge University Press, New York, 1998).

[3] E. Ben-Jacob, R. Godbey, N. D. Goldenfeld, J. Koplik, H. Levine, T. Mueller, and L. M. Sander, Phys. Rev. Lett. 55, 1315 (1985).

[4] J.-D. Chen and D. Wilkinson, Phys. Rev. Lett. 55, 1892 (1985).

[5] V. Horváth, T. Vicsek, and J. Kértesz, Phys. Rev. A 35, 2353 (1987).

[6] J.-D. Chen, Exp. Fluids 5, 363 (1987).

[7] E. Ben-Jacob, P. Garik, T. Mueller, and D. Grier, Phys. Rev. A 38, 1370 (1988).

[8] J. Ignés-Mullol and J. V. Maher, Phys. Rev. E 53, 3788
(1996).

[9] P. Falus and V. K. Horváth (unpublished).

[10] F. Liu and N. Goldenfeld, Phys. Rev. A 42, 895 (1990).

[11] R. Almgren, W.-S. Dai, and V. Hakim, Phys. Rev. Lett. 71, 3461 (1993).

[12] A. G. Banpurkar, A. S. Ogale, and A. V. Limaye, Phys. Rev. E 59, 2188 (1999).

[13] S. N. Rauseo, P. D. Barnes, Jr., and J. V. Maher, Phys. Rev. A 35, 1245 (1987).

[14] S. K. Sarkar and D. Jasnow, Pramana, J. Phys. 41, 389 (1993).

[15] S. K. Sarkar and D. Jasnow, Phys. Rev. A 39, 5299 (1989).

[16] A. M. Lacasta, L. Ramírez-Piscina, J. Casademunt, A. Hernández-Machado, and M. A. Rodríguez, Phys. Rev. E 57, 5754 (1998). 